\section{Efeitos de diferentes intensidades de trein- amento físico aeróbio sobre a concentração cir- culante de leucócitos em adolescentes obesos submetidos à intervenção multidisciplinar}

\author{
Effects of different aerobic training intensi- \\ ty on leukocyte in obese adolescents sub- \\ mitted to a multidisciplinary therapy
}

\author{
Thiago Ricardo dos Santos Tenório ${ }^{1,2}$ \\ Camila Tenório Calazans de Lira ${ }^{1}$ \\ Humberto José Gomes da Silva ${ }^{1}$ \\ Mara Cristina Lofrano-Prado \\ Moacir Novaes de Lima Ferreira ${ }^{4}$ \\ Sérgio Luiz Cahú Rodrigues ${ }^{1}$ \\ Wagner Luiz do Prado ${ }^{1,2}$
}

\section{Resumo}

O objetivo do presente estudo foi comparar os efeitos do treinamento aeróbio de alta intensidade (TAI) com o de baixa intensidade (TBI) sobre a concentração circulante de leucócitos em adolescentes obesos. Quarenta e três adolescentes obesos $\left(34,4 \pm 4,3 \mathrm{~kg} / \mathrm{m}^{2}\right)$ de ambos os gêneros (13 meninos e 30 meninas), com idade entre 13 e 18 anos, foram submetidos de forma aleatória ao TAI (intensidade correspondente ao limiar ventilatório I; N=20) ou TBI (intensidade correspondente a 20\% abaixo do limiar ventilatório I; N=23) durante 12 semanas. Todos os voluntários receberam o mesmo acompanhamento clínico, psicológico e nutricional. Para ambos os grupos as sessões de exercício foram isocalóricas (350Kcal). A concentração total de leucócitos e subpopulações (monócitos, linfócitos e neutrófilos) foram determinadas por citometria de fluxo fluorescente antes (pré) e após (pós) a intervenção. Para a análise entre grupos e momentos foi utilizada ANOVA two way para medidas repetidas, com post-hoc de Fisher. Correlações entre as variáveis foram analisadas por meio da correlação de Pearson. Os resultados demonstraram redução na concentração circulante de monócitos tanto no TAI (pré: $0,63 \pm 0,10$; pós: $0,3 \pm 0,10-p<0,001$ ) quanto no TBI (pré: $0,73 \pm 0,27$; pós: $0,39 \pm 0,19-p<0,001$ ), sem diferença entre os grupos. Adicionalmente o foi verificado elevação dos neutrófilos apenas no grupo TAI ( $\mathrm{p}=0,008)$. Dessa forma, podemos concluir que treinamento físico aeróbio, independente da intensidade, é efetivo no controle do estado inflamatório em adolescentes obesos, via redução da concentração circulante de monócitos.

\section{Palavras-chave}

Exercício físico; Inflamação; Obesidade; Adolescência.

\begin{abstract}
The aim of this study was to compare the effects of a high intensity (HIT) vs. a low intensity (LIT) aerobic exercise training on leukocytes levels in obese adolescents. Forty-three obese $\left(34.4 \pm 4.3 \mathrm{~kg} / \mathrm{m}^{2}\right)$ from both genders (13 boys and 30 girls) aged between 13 and 18 years underwent randomically to HIT (intensity corresponding to ventilatory threshold I, N=20) or LIT (intensity corresponding to $20 \%$ below ventilatory threshold I, N=23) during 12 weeks. All volunteers accomplish the clinical follow-up (1x/month), psychological and nutritional counseling $(1 x /$ week). For both groups exercise sessions were isocaloric (150Kcal). Total leukocytes and subpopulations (monocytes, lymphocytes and neutrophils) were determined by fluorescence flow cytometry before and after intervention. For analysis between groups and time were used two-way ANOVA for repeated measures with Fisher as post-hoc. Correlations between variables were analyzed by Pearson's correlation. The results showed reduction on circulating levels of monocytes in TAI (pre: $0.63 \pm 0.10$; post: $0.3 \pm 0.10-p<0.001$ ) as well as in TBI (pre: $0.73 \pm 0.27$; post: $0.39 \pm 0.19-p<0.001)$, without groups differences. In addition it was verified a rise in neutrophils values ( $p=0.008)$. Thus we can conclude that aerobic exercise, regardless of intensity, is effective in controlling the inflammatory status in obese adolescents by reducing monocytes circulating levels.
\end{abstract}

\section{Keywords}

Exercise; Inflammation; Obesity; Adolescence.
Rev Bras Ativ Fis Saúde p. 414-422 DOI: http://dx.doi.org/10.12820/23171634.2012v17n5p414

1 Escola Superior de Educação Física, Universidade de Pernambuco, Recife, PE, Brasil.

2 Programa Associado de Pós-graduação em Educação Física Universidade de Pernambuco/Universidade da Paraíba, Recife, $P E$, Brasil.

3 Programa de Pós-graduação em Nutrição, Universidade Federal de Pernambuco, Recife, PE, Brasil.

4 Faculdade de Medicina, Universidade de Pernambuco, Recife, PE, Brasil. 


\section{INTRODUÇÃO}

A obesidade é reconhecida como um potente fator de risco para o desenvolvimento de doenças cardiovasculares (DCV $)^{(1)}$. Acredita-se que a gênese dos sintomas clínicos das DCV inicia-se ainda na infância e tornam-se mais aparentes em períodos tardios da vida ${ }^{(2)}$. Entretanto, os riscos da obesidade em crianças e adolescentes, não são limitados a complicações na vida adulta ${ }^{(3)}$, visto que crianças obesas já apresentam alterações nos componentes da síndrome metabólica (SM), comumente observadas apenas em adultos ${ }^{(4)}$.

Além dos efeitos negativos diretos do excesso de gordura corporal na saúde do indivíduo, a obesidade está associada a alterações nas concentrações circulantes de marcadores pró e antiinflamatórios, o que faz com que o obeso apresente um quadro de inflamação crônica subclínica ${ }^{(5)}$. Este estado inflamatório crônico eleva o risco para o desenvolvimento de diversas condições deletérias à saúde, tais como resistência à ação da insulina, problemas cardiovasculares, hipertensão arterial sistêmica, dislipidemia aterogênica, disfunção endotelial ${ }^{(7)}$ entre outras.

Técnicas sofisticadas de análises bioquímicas são utilizadas em pesquisas científicas para o monitoramento do estado inflamatório de obesos e não obesos, como por exemplo, a quantificação da concentração circulante do fator de necrose tumoral alfa (TNF- $\alpha$ ), de interleucinas (ex: IL-1 e IL-6), proteína c-reativa (PCR), fibrinogênio entre outros. No entanto, tais técnicas apresentam elevado custo e pouca aplicabilidade na prática clínica. Desta forma, a contagem de leucócitos e subpopulações constituem um marcador de baixo custo, confiável, de fácil interpretação e rotineiramente utilizado como indicador da resposta imunológica ${ }^{(8)}$, sendo o seu monitoramento uma alternativa para o acompanhamento de quadros inflamatórios ${ }^{(8)}$.

Adicionalmente, a elevação da concentração total de células brancas (leucócitos, neutrófilos, linfócitos e monócitos), é um fator de risco independente de morbidade e mortalidade por doença coronariana em todos os indivíduos ${ }^{(9)}$.

Programas de intervenção multidisciplinares que incluam orientação nutricional, clínica e psicológica, além da prática regular de exercícios físicos são os mais indicados para o tratamento de pacientes obesos, e tais intervenções são mais efetivas em crianças e adolescentes do que em adultos ${ }^{(10)}$. Neste contexto, o exercício físico ocupa um lugar de destaque, pois além de auxiliar o controle ponderal, é um recurso efetivo e seguro no gerenciamento dos riscos cardiometabólicos associados à obesidade ${ }^{(11)}$.

Estudos prévios demonstraram que o treinamento físico, especialmente o aeróbio, promove alterações benéficas no perfil lipídico ${ }^{(12)}$, glicídico ${ }^{(13)}$, composição corporal $^{(14)}$, além de melhorar a aptidão física ${ }^{(15)}$ e qualidade de vida ${ }^{(16)}$ de obesos, entretanto, vale ressaltar que os efeitos do treinamento aeróbio são intensidadedependente, e até o momento são escassos os estudos que analisaram os efeitos de diferentes intensidades de treinamento aeróbio sobre o perfil inflamatório de adolescentes obesos.

Dada a relevância do tema e a necessidade eminente do desenvolvimento de tratamentos mais eficazes no gerenciamento das desordens cardiometabólicas associadas à obesidade, o presente estudo objetivou comparar os efeitos do treinamento aeróbio de alta intensidade com o de baixa intensidade sobre a concentração circulante de leucóctios e subpopulações em adolescentes obesos submetidos à intervenção multidisciplinar. 


\section{MÉTODOS}

Os Adolescentes obesos foram recrutados entre Janeiro e Fevereiro de 2011 através de divulgação em rádio, televisão e jornais regionais. Os critérios de inclusão adotados foram idade entre 13 e 18 anos, púberes (estágio 3 e 4 de Tanner), massa corporal inferior a $120 \mathrm{~kg}$, índice de massa corporal (IMC) acima do percentil 95 da curva de Must e cols. ${ }^{(17)}$, ausência de hipertensão e/ou outras doenças metabólicas, aptos a prática regular de exercícios físicos e motivação intrínseca para participar da intervenção multidisciplinar para tratamento da obesidade por 12 semanas.

Após serem esclarecidos sobre todos os procedimentos experimentais, os pais ou responsáveis legais assinaram Termo de Consentimento Livre e Esclarecido. Este estudo foi aprovado pelo Comitê de Ética da Universidade de Pernambuco (n¹54/09). A pesquisa foi conduzida dentro dos padrões éticos exigidos pela Declaração de Helsinque de 1964 e de acordo com a resolução 196/96 do Ministério da Saúde.

Inicialmente, 229 adolescentes se voluntariam a participar do estudo. Durante a primeira visita foram realizadas as medidas antropométricas (massa corporal e estatura), determinação do estágio maturacional e os adolescentes foram submetidos a uma entrevista semi-estruturada para verificar a motivação intrínseca. As entrevistas eram compostas por 8 questões relacionadas a motivos e barreiras para perder peso, e foram conduzidas pela psicóloga do grupo. Cento e oitenta e seis voluntários não atenderam aos critérios de inclusão. Na segunda visita ao laboratório, os adolescentes foram submetidos à consulta clínica e realizaram eletrocardiograma de esforço e repouso. Ao final, 43 adolescentes obesos (13 meninos e 30 meninas) foram incluídos na amostra. Após as avaliações basais, os participantes foram aleatoriamente alocados no grupo treinamento de alta intensidade (TAI) $(n=20)$ ou grupo treinamento de baixa intensidade (TBI) $(\mathrm{N}=23)$. Todos os adolescentes receberam a mesma intervenção nutricional, psicológica e clínica.

\section{Intervenção clínica}

A partir da primeira consulta com o endocrinologista responsável pelo projeto, os adolescentes recebiam acompanhamento clínico mensal. Durante as consultas o endocrinologista orientava sobre mudanças no estilo de vida, aferia a pressão arterial e verificava a evolução dos voluntários por meio dos resultados dos exames clínicos e laboratoriais. Não houve prescrição de nenhum tipo de remédio que pudesse de alguma forma alterar os resultados da pesquisa.

\section{Intervenção nutricional}

A intervenção nutricional foi composta por encontros em grupos (aproximadamente 09 adolescentes), uma vez por semana, com duração de uma hora. As atividades sempre foram desenvolvidas e supervisionadas pela nutricionista do grupo. Durante os encontros eram abordados temas como pirâmide alimentar, fastfood, rotulagem nutricional, dietas da moda, nutrição na promoção de saúde, tipos de gordura (trans, saturada e insaturada), produtos diet vs light, comportamento em ocasiões especiais (festas, aniversários, eventos sociais). Não foi prescrita nenhuma dieta individualizada.

\section{Intervenção psicológica}

Foram realizadas sessões semanais de terapia em grupo (aproximadamente 09 adolescentes), com uma hora de duração, conduzidas pela psicóloga do grupo. Du- 
rante as sessões foram abordados temas como emoções (sentimentos), auto-estima, imagem corporal, preconceito, transtornos alimentares, dificuldades, questões familiares, bem como questões que pudessem surgir no grupo. Além disso, a psicóloga ficava à disposição dos voluntários para apoio e acolhimento, se necessário.

\section{Treinamento Aeróbio}

Os participantes de ambos os grupos realizaram treinamento aeróbio individualizado em esteira rolante, 3 vezes por semanas, sob a supervisão de um profissional de educação física. Os voluntários do TAI treinaram em esteira rolante em velocidade $(\mathrm{Km} / \mathrm{h})$ correspondente ao limiar ventilatório I (LVI); os do TBI exercitaram-se em velocidade $(\mathrm{Km} / \mathrm{h})$ correspondente a $20 \%$ abaixo do LVI. Para ambos os grupos as sessões foram isocalóricas, com o gasto energético fixado em $350 \mathrm{Kcal} /$ sessão, estimado por calorimetria indireta. Uma vez que a intensidade de treinamento era individualizada, a duração das sessões diferiu entre os sujeitos. Para garantir que o gasto energético pré-fixado fosse atingido, a duração de cada sessão foi determinada considerando-se que para cada litro de oxigênio consumido $\left(\mathrm{VO}_{2}\right.$ $\mathrm{L} / \mathrm{min}$ ), 4,96 kcal de energia era liberada, baseado no equivalente metabólico da tarefa (MET), conforme a seguir:

Duração da sessão de exercício $(\mathrm{min})=350 \mathrm{Kcal} /\left(\mathrm{VO}_{2}(\mathrm{~L} / \mathrm{min})\right.$ na carga $\left.* 1 \mathrm{MET}\right)$

Onde: $\mathrm{VO}_{2}=$ consumo de oxigênio; $\mathrm{MET}=$ equivalente metabólico da tarefa.

\section{Avaliações}

Todos os voluntários foram submetidos ao mesmo protocolo de avaliações, antes (pré) e após 12 semanas (pós) de intervenção multidisciplinar. As medidas eram realizadas sempre no mesmo período do dia, com o objetivo de se minimizar quaisquer variações circadianas nas variáveis analisadas.

\section{Medidas antropométricas e composição corporal}

As medidas de massa corporal (MC), estatura e circunferência de cintura (CC) foram realizadas em uma balança Filizola ${ }^{\circledR}$ com precisão de $0,1 \mathrm{~kg}$, um estadiômetro de madeira com precisão de $0,1 \mathrm{~cm}$ e fita métrica inextensível com precisão de $0,1 \mathrm{~cm}$, respectivamente, adotando os critérios propostos por Lohman e cols. ${ }^{(18)}$. Em seguida foi calculado o índice de massa corporal (IMC), dividindo-se a massa corporal $(\mathrm{Kg})$ pelo quadrado da estatura $\left(\mathrm{m}^{2}\right)$.

As dobras cutâneas triciptal (TR), subescapular (SE) e da panturrilha medial (PA) foram mensuradas com um adipômetro de marca Lange $e^{\circledR}$ com pressão constante de $\left(10 \mathrm{~g} / \mathrm{mm}^{2}\right)$ e resolução de $1 \mathrm{~mm}$, no hemicorpo direito. Após três medidas, a média era calculada. Todas as mensurações seguiram a padronização sugerida por Lohman e cols. ${ }^{(18)}$. O percentual de gordura $(\% \mathrm{G})$ foi estimado através da equação de Slaughter e cols. ${ }^{(19)}$.

\section{Consumo de Oxigênio e determinação do limiar ventilatório}

$\mathrm{O} \mathrm{VO}_{2}$ foi determinado através de análise direta de gases, utilizando-se um analisador metabólico de circuito aberto (Quark PFT, Cosmed, Italy), durante teste contínuo e incremental em esteira rolante (Cosmed T200, Italy). Após aquecimento de 3 minutos a $3 \mathrm{Km} / \mathrm{h}$, a velocidade era acrescida em $1 \mathrm{Km} / \mathrm{h}$ a cada minuto até a exaustão voluntária, ou quando a escala de Borg e o quociente respiratório apresentassem valores superiores a 18 e 1,15, respectivamente, a inclinação foi 
mantida constante em $1 \%$ durante todo o teste. $\mathrm{O}$ maior $\mathrm{VO}_{2}$ obtido antes da interrupção do teste foi considerado como consumo pico de oxigênio $\left(\mathrm{VO}_{\text {2pico }}\right)$. Antes de cada teste, os instrumentos eram calibrados para a mistura de gases $\left(\mathrm{O}_{2}\right.$ $=12,2$ e $\mathrm{CO}_{2}=4,8-$ White Martins) e volume, seguindo-se todas as instruções do fabricante.

O LVI foi determinado por meio de inspeção visual por dois pesquisadores independentes, como o ponto de quebra de linearidade entre a produção de dióxido de carbono $\left(\mathrm{CO}_{2}\right)$ e o $\mathrm{VO}_{2}$ (V-slope); ponto no qual a curva do equivalente ventilatório de oxigênio $\left(\mathrm{VE} / \mathrm{VO}_{2}\right)$ e a fração expirada de $\mathrm{CO}_{2}\left(\mathrm{P}_{\mathrm{ET}} \mathrm{CO}_{2}\right)$ atingiram seus menores valores, imediatamente antes do aumento dos respectivos equivalentes $^{(20)}$.

\section{Análises bioquímicas}

A coleta de sangue foi realizada através de punção periférica na veia cubital, entre às 8:00 e 9:00h da manhã, após jejum noturno de 12 horas, em seguida as alíquotas foram estocadas em tubos com anticoagulante (EDTA-K3E). A contagem total de leucócitos e subpopulações (monócitos, linfócitos e neutrófilos) foram determinadas pelo método de citometria de fluxo fluorescente (Analisador hematológico automatizado - Sysmex XE 2100 ${ }^{T M}$ ).

\section{Análise estatística}

Os dados foram analisados através do programa SPSS versão 20 e Statistica versão 8.0. A normalidade dos dados foi determinada através do teste de Shapiro Wilk, e os dados são apresentados como média \pm desvio padrão. Para a análise comparativa entre os grupos (TAI e TBI) e os momentos (pré e pós) foi utilizada a análise de variância de dois caminhos para medidas repetidas (ANOVA - two way), com Post -hoc de Fisher. Utilizou-se o teste de correlação de Pearson para verificar possíveis associações entre os deltas de variação, entre a $\mathrm{MC}, \% \mathrm{G}, \mathrm{IMC}$ e contagem total de leucócitos, monócitos, neutrófilos e linfócitos. Adicionalmente, foi calculado o effect size intra-grupo, sendo o tamanho do efeito expresso como correlação quando o efeito foi maior que moderado ( $r>0,3)$, conforme proposto por Cohen ${ }^{(21)}$.

\section{RESULTADOS}

A amostra inicial foi composta por 43 adolescentes (13 meninos e 30 meninas; $15,4 \pm 1,5$ anos; $\left.34,4 \pm 4,3 \mathrm{~kg} / \mathrm{m}^{2}\right)$, entretanto sete voluntários foram excluídos, três do TAI (01 por problemas ortopédicos e 02 por aderência inferior a $75 \%$ ) e quatro do TBI (aderência inferior a 75\%). Dessa forma ao final das 12 semanas de intervenção, a taxa de adesão foi de $85 \%$ para o TAI (06 meninos e 11 meninas) e de $83 \%$ para o TBI (12 meninas e 07 meninos), sem diferenças entres os grupos. As sessões de TBI foram mais longas $(57,1 \pm 11,71 \mathrm{~min}$. $)$ do que TAI $(40,7 \pm 8,71$ $\min ).(\mathrm{p}<0,000)$.

Em resposta à intervenção multidisciplinar, foram verificadas reduções na massa corporal $(\mathrm{p}<0,001)$, IMC $(\mathrm{p}<0,001)$, massa gorda $(\mathrm{p}<0,001)$, circunferência da cintura $(p<0,001)$, percentual de gordura $(p<0,001)$ e somatório das dobras cutâneas $(\mathrm{p}<0,001)$ e aumento do $\mathrm{VO}_{2 \text { pico }}(\mathrm{p}<0,001)$ em ambos os grupos; adicionalmente houve incrementos na massa magra $(\mathrm{Kg})$, para os adolescentes que treinaram em ambos os grupos $(\mathrm{p}<0,001)($ tabela 1$)$. 
Tabela 1 - Efeitos de diferentes intensidades de treinamento físico aeróbio sobre variáveis antropométricas e composição corporal de adolescentes obesos submetidos à intervenção multidisciplinar.

\begin{tabular}{|c|c|c|c|c|}
\hline & \multicolumn{2}{|c|}{ TAI $(N=17)$} & \multicolumn{2}{|c|}{ TBI $(\mathrm{N}=19)$} \\
\hline & Pré & Pós & Pré & Pós \\
\hline Meninas/Meninos & \multicolumn{2}{|c|}{$11 / 6$} & \multicolumn{2}{|c|}{$12 / 07$} \\
\hline Idade (anos) & $15,77 \pm 1,34$ & $16,10 \pm 1,34$ & $15,34 \pm 1,63$ & $15,66 \pm 1,63$ \\
\hline Estatura (m) & $1,62 \pm 0,05$ & $1,62 \pm 0,05$ & $1,62 \pm 0,08$ & $1,63 \pm 0,09$ \\
\hline$M C(k g)$ & $90,31 \pm 11,27$ & $87,75 \pm 11,73^{*}$ & $92,11 \pm 11,41$ & $90,42 \pm 12,39 *$ \\
\hline $\mathrm{IMC}(\mathrm{kg} / \mathrm{m} 2)$ & $34,30 \pm 4,12$ & $33,43 \pm 4,03^{*}$ & $34,64 \pm 3,75$ & $33,76 \pm 3,93^{*}$ \\
\hline Cintura $(\mathrm{cm})$ & $96,3 \pm 8,9$ & $92,8 \pm 8,0^{*}$ & $94,6 \pm 6,7$ & $92,5 \pm 8,4^{*}$ \\
\hline Gordura(\%) & $49,92,0 \pm 6,99$ & $40,45 \pm 9,78^{*}$ & $53,13 \pm 9,39$ & $38,07 \pm 7,08^{*}$ \\
\hline M.Gorda(Kg) & $46,3 \pm 9,30$ & $40,0 \pm 13,0^{*}$ & $50,1 \pm 13,8$ & $37,6 \pm 9,60^{*}$ \\
\hline M.Magra $(\mathrm{Kg})$ & $50,07 \pm 6,99$ & $59,54 \pm 9,78^{*}$ & $43,11 \pm 6,34$ & $55,29 \pm 9,20^{*}$ \\
\hline$\Sigma \mathrm{DcTR} / \mathrm{SE}(\mathrm{mm})$ & $71,9 \pm 10,0$ & $61,0 \pm 14,4^{*}$ & $74,0 \pm 14,0$ & $56,1 \pm 11,4^{*}$ \\
\hline VO2pico (ml.kg.min-1) & $25,6 \pm 4,5$ & $30,1 \pm 4,5^{*}$ & $26,5 \pm 5,2$ & $30,0 \pm 5,6^{*}$ \\
\hline
\end{tabular}

$M C=$ massa corporal; $I M C=$ índice de massa corporal; Cintura=circunferência da cintura; $M$.Gorda=massa gorda; M.Magra=massa magra; $\Sigma \mathrm{DcTR} / \mathrm{SE}=$ somatório da dobra cutânea tricipital e subescapular; $\mathrm{VO}_{2 \text { pico }}=$ consumo pico de oxigênio $\mathrm{TAI}=$ treinamento de alta intensidade; $\mathrm{TBI}=$ treinamento de baixa intensidade. *Pré $x$ Pós $(p<0,001)$ ANOVA Two Way

A tabela 2 demonstra que, independente da intensidade de treinamento aeróbio, a intervenção multidisciplinar não modulou os valores de leucócitos e linfócitos, entretanto o TAI promoveu redução na concentração circulante de monócitos $(\mathrm{p}<0,001)$ e elevação de neutrófilos $(\mathrm{p}=0,008)$, enquanto que o TBI apenas alterou os valores de monócitos $(\mathrm{p}<0,001)$. Embora ambas as intensidades tenham sido efetivas na redução de monócitos, o effectsize foi alto para o TAI $(\mathrm{r}=0,82)$ e moderado para o TBI $(r=0,57)$. Para nenhuma das variáveis estudadas houve diferença entre os grupos nos momentos pré e pós intervenção. Adicionalmente, não foram verificadas correlações entre as alterações antropométricas e de composição corporal com os valores de leucócitos e subpopulações.

Tabela 2 - Efeitos de diferentes intensidades de treinamento físico aeróbio sobre a concentração circulante de leucócitos e subpopulações em adolescentes obesos submetidos à intervenção multidisciplinar.

\begin{tabular}{lcccc}
\hline & \multicolumn{2}{c}{ TAI $(\mathrm{N}=17)$} & \multicolumn{2}{c}{ TBI $(\mathrm{N}=19)$} \\
\hline & Pré & Pós & Pré & Pós \\
\hline Leucócitos (103/uL) & $7,95 \pm 1,60$ & $8,33 \pm 2,14$ & $8,50 \pm 2,10$ & $8,29 \pm 2,56$ \\
Neutrófilos (103/uL) & $4,29 \pm 1,35$ & $4,97 \pm 1,97^{*}$ & $4,78 \pm 1,92$ & $4,72 \pm 2,01$ \\
Linfócitos (103/uL) & $2,63 \pm 0,43$ & $2,76 \pm 0,58$ & $2,72 \pm 0,45$ & $2,83 \pm 0,60$ \\
\hline Monócitos (103/uL) & $0,63 \pm 0,12$ & $0,31 \pm 0,10^{*}$ & $0,73 \pm 0,27$ & $0,39 \pm 0,19 *$ \\
\hline
\end{tabular}

$\mathrm{GTAl}=$ Grupo tratamento alta intensidade; GTBI=Grupo tratamento baixa intensidade. ${ }^{*}$ Pré $\mathrm{x}$ Pós $(p<0,05)$ ANOVA Two Way.

\section{DISCUSSÃO}

Estudos demonstram que elevada concentração circulante de leucócitos, como observada em pacientes com inflamação crônica, constitui um fator de risco independente para o desenvolvimento de doenças cardiovasculares ${ }^{(8)}$, e dentre as subpopulações leucocitárias, os monócitos são descritos como o tipo celular predominante no perfil inflamatório em processos ateroscleróticos ${ }^{(22)}$. Desta forma, o principal achado do presente estudo é que o treinamento aeróbio, independente 
da intensidade, é capaz de reduzir a concentração circulante de monócitos em adolescentes obesos.

O perfil leucocitário encontra-se positivamente associado com IMC, percentual de gordura e circunferência da cintura ${ }^{(23)}$, neste sentido, foi verificado que obesos submetidos a cirurgia bariátrica apresentaram redução da contagem total de leucócitos quando comparados a obesos que não participaram de nenhuma intervenção para controle de peso ${ }^{(24)}$, demonstrando que a redução da massa corporal total e da adiposidade em obesos, constituem uma importante via no controle do estado inflamatório, independente de mudanças no estilo de vida (dieta e prática de atividade física).

Entretanto, no presente estudo, reduções na massa corporal e nos indicadores de adiposidade não apresentaram associação com as alterações no perfil leucocitário. Em um recente estudo, Lamina e Okoye ${ }^{(25)}$, demonstraram que homens hipertensos e com sobrepeso, após oito semanas de treinamento físico em cicloergômetro, com intensidade moderada (35-60\% da freqüência cardíaca máxima de reserva) e com duração de 60 minutos, apresentaram diminuição significativa na contagem total de leucócitos, sem alterações no IMC. Ainda nesse sentido, Michishita e cols. (26), em um estudo conduzido com mulheres com sobrepeso, encontraram diminuição na concentração total de leucócitos após 6 semanas de treinamento aeróbio (intensidade correspondente ao limiar de lactato). Johannse e cols. ${ }^{(27)}$, após submeterem mulheres obesas a 24 semanas de treinamento aeróbio de baixa intensidade $\left(50 \% \mathrm{VO}_{2 \text { pico }}\right)$, relataram diminuição da contagem de leucócitos e subpopulações independente de alterações em indicadores de adiposidade. Tais achados, juntamente com os reportados no presente estudo, reforçam o potencial antiinflamatório do treinamento físico aeróbio, mesmo na ausência de alterações na massa corporal.

Três possíveis mecanismos tem sido sugeridos para explicar o efeito antiinflamatório do treinamento físico: 1) diminuição da concentração circulante de adipocinas pró-inflamatórias em consequência da redução da adiposidade visceral; 2) aumento na produção e secreção de IL-6 proveniente da contração muscular, o que estimula a ativação de respostas antiinflamatórias; e 3) redução da expressão de receptores do tipo Toll-like sobre monócitos e macrófagos ${ }^{(28)}$,o que contribui para o controle imunitário. Desta forma, sugerimos que a diminuição da contagem de monócitos, encontrada no presente estudo, pode ter sido ser influenciada pela melhoria da função do músculo esquelético e da função endotelial ${ }^{(26)}$ dos adolescentes.

Por outro lado, verificamos no presente estudo elevação da contagem de neutrófilos em resposta ao treinamento de alta intensidade, o que poderia facilitar a instalação de processos inflamatórios. Infelizmente, não existem estudos na literatura que nos permitam formular hipóteses para explicar tal achado. Vale ressaltar que, mesmo após a intervenção, os valores de neutrófilos encontravam-se dentro dos valores de normalidade.

O presente estudo apresenta algumas limitações que devem ser consideradas para a interpretação e aplicação dos resultados, sendo necessários mais estudos que avaliem além da concentração circulante das células brancas, a sua função e ativação, através da dosagem de marcadores enzimáticos específicos (mieloperoxidase e elastase, por exemplo), bem como a utilização de técnicas mais precisas para estimativa da composição corporal e da gordura visceral, tais como a densitometria por duplo feixe (DEXA) e ultrassonografia.

Vale ressaltar que no presente estudo a determinação da intensidade do exercício físico foi baseada no limiar ventilatório o que minimiza diferenças individuais, 
comumente observadas quando a intensidade do esforço é relativa ao consumo máximo de oxigênio $\left(\mathrm{VO}_{2 \max }\right)$ ou percentual da freqüência cardíaca máxima.

Os resultados do presente estudo reforçam a necessidade da prática regular de atividade física por adolescentes obesos, visando não apenas o controle da adiposidade, mas também a redução de possíveis complicações associadas à obesidade na vida adulta, uma vez que, o exercício físico promove proteção contra diversas causas de mortalidade e morbidade associadas a processos inflamatórios crônicos ${ }^{(29)}$.

Desta forma, podemos concluir que, o treinamento físico aeróbio é eficiente no controle do estado inflamatório crônico observado em adolescentes obesos, principalmente via redução da concentração circulante de monócitos, e que tais resultados são independentes da intensidade do treinamento.

\section{Agradecimentos}

Os autores agradecem ao Conselho Nacional de Desenvolvimento Científico e Tecnológico (CNPq), a Fundação de Apoio à Pesquisa e Tecnologia do Estado de Pernambuco (FACEPE) e ao Programa de Fortalecimento Acadêmico da UPE. Especialmente a todos os voluntários.

\section{REFERÊNCIAS}

1. Zalesin KC, Franklin BA, Miller WM, Peterson ED, McCullough PA. Impact of obesity on cardiovascular disease. Med Clin North Am 2011;95:919-37.

2. Reilly JJ, Kelly J. Long-term impact of overweight and obesity in childhood and adolescence on morbidity and premature mortality in adulthood: systemic review. Int J Obes (Lond) 2011;35: 891-8.

3. Weiss R, Dziura J, BurgetTS, et al. Obesity and metabolic syndrome in children and adolescents. N Engl J Med 2004;350:2362-74.

4. McCall A, Raj R. Exercise for prevention of obesity and diabetes in children and adolescents. Clin Sports Med 2009;28:393-421.

5. Dalmas E, Roualt C, Abdennour M, et al. Variations in circulating inflammatory factors are related to changes in calorie and carbohydrate intekes early in the course of sugery-induced weight reduction. Am J ClinNutr 2011;94:450-8.

6. Kong AP, Chow CC. Medical consequences of childhood obesity: a Hong Kong perspective. Res Sports Med 2010;18:16-25.

7. Coller BS. Leukocytosis and ischemic vascular disease morbidity and mortality: is it time to intervene? ArteriosclerThrombVascBiol 2005;25:658-70.

8. Engström G, Melander O, Hedblad B. Leukocyte count and incidence of hospitalizations due to heart failure. Circ Heart Fail 2009; 2:217-22.

9. Gillum RF, Mussolino ME, MadansJH. Counts of neutrophils, lyphocytes, and monocytes, cause-specific mortality and coronary heart disease: the NHANES-I epidemiologic follow-up study. Ann Epidemiol 2005;15:266-7.

10. Snethen JA, Broome ME, Cashin SE. Effective weight loss for overweight children: a metaanalysis of intervention studies. J PediatrNurs 2006;21:45-56.

11. Matus CD, Klaege K. Exercise and weight management. Prim Care 2007;34:109-16.

12. Kraus WE, Houmard JA, Duscha BD, et al. Effects of the amount and intensity of exercise on plasma lipoprotein. N Eng1 J Med 2002;347:1483-92.

13. Shaw K, Gennat H, O Rourke P, Del Mar C. Exercise for overweight or obesity. Cochrane Database Syst Rev 2006; 18(4).

14. Lazzer S, Lafortuna C, Busti C, et al. Effects of low-and high-intensity exercise training on body composition and substrate metabolism in obese adolescents. J Endocrinol Invest 2011;34:45-52.

15. Buchan DS, Ollis S, Thomas NE, et al. Physical activity interventions: effects of duration and intensity. Scand J Med Sci Sports 2011;21:341-50. 
16. Lofrano-Prado MC, Antunes HK, do Prado WL, et al. Quality of life in Brazilian obese adolescents: effects of a long-term multidisciplinary lifestyle therapy. Health Qual Life Outcomes 2009;7:1-8.

17. Must A, Dallal GE, Dietz WH. Reference data for obesity: 85 th and 95 th percentiles of body mass index (wt/ht2) and triceps skinfold thickness. Am J ClinNutr 1991;54:839-46.

18. Lohman TG, Roche AF, Martorell R. Anthropometric satandardizitions reference manual. Champaign: Human Kinectics Books, 1988.

19. Slaughter MH, Lohman TG, Boileau RA, et al. Skinfold equations for estimation of body fatness in children and youth. Hum Biol 1988:60:709-23.

20. Wasserman K. The anaerobic threshold measurement to evaluate exercise performance. Am Rev Respir Dis 1984;129:S35-40.

21. Cohen J. Statistical power analysis for the behavioral sciences. New York: Lawrence Erlbaum Associates, 1977.

22. Atmaca Y, Ozdol C, Turhan S, et al. The association of elevated white blood cell count and c-reactive protein with endothelial dysfunction in cardiac syndrome x. ActaCrdiol 2008;63:723-8.

23. KulloIJ, Hensrud DD, Allison TG. Comparison of numbers of circulating blood monocytes in men grouped by body mass index $(<25,25$ to $<30,>$ or $=30)$. Am J Cardiol2002;89:1441-3.

24. Veronelli A, Laneri M, Ranieri R, et al. White blood cells in obesity and diabetes: effects of weight loss and normalization of glucose metabolism. Diabetes Care 2004;27:2501-2.

25. Lamina S, Okoye CG. Effect of interval training programo on white blood cell count in the management of hypertension: a randomized controlled study. Niger Med 2011;52:271-77.

26. Michishita R, Shono N, Inoue T, Tsuruta T, Node K. Effect of exercise therapy on monocyte and neutrophil counts in overweight women. Am J Med Sci 2010;339:152-6.

27. Johannsen NM, Swift DL, Johnson WD, et al. Effect of different doses of aerobic exercise on total white blood cell (WBC) and WBC subfraction number in postmenopausal women: results from DREW. PLoS One 2012;7:e31319.

28. Flynn MG, McFarlin BK. Toll-like receptor 4: link to the anti-inflammatory effects of exercise?Exerc Sport Sci Rev 2006;34:176-81.

29. Mathur N, Pedersen BK. Exercise as a mean to control low-grade systemic inflammation. Mediators Inflamm 2008;2008:109502.

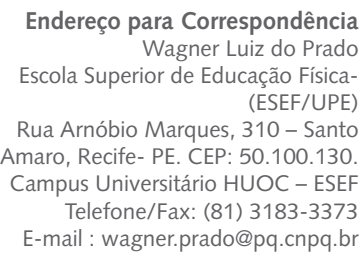

Recebido 23/10/2012

Revisado 02/01/2013

Aprovado 05/01/2013 